\title{
PERSPECTIVES
}

SCIENCE \& SOCIETY

\section{The X-files in immunity: sex-based differences predispose immune responses}

\section{Eleanor N. Fish}

Abstract | Despite accumulating evidence in support of sex-based differences in innate and adaptive immune responses, in the susceptibility to infectious diseases and in the prevalence of autoimmune diseases, health research and clinical practice do not address these distinctions, and most research studies of immune responses do not stratify by sex. X-linked genes, hormones and societal context are among the many factors that contribute to disparate immune responses in males and females. It is crucial to address sex-based differences in disease pathogenesis and in the pharmacokinetics and pharmacodynamics of therapeutic medications to provide optimal disease management for both sexes.
Human illnesses affect men and women differently. In general, both the proportion of individuals infected and the severity of infection are higher in males than females for viral, bacterial, fungal and parasitic diseases ${ }^{1,2}$. However, the prevalence of sexually transmitted infections (STIs), such as HIV and herpes simplex virus-2 (HSV-2), is higher among women. In addition, many autoimmune diseases affect more women than men; systemic lupus erythematosus (SLE), Graves' disease, Hashimoto's thyroiditis and Sjögren's syndrome exhibit a 7-10:1 female:male predominance, and multiple sclerosis, rheumatoid arthritis and scleroderma a 2-3:1 female:male ratio. By contrast, ankylosing spondylitis, Goodpasture syndrome, Reiter syndrome and vasculitis all occur predominantly in males ${ }^{3,4}$.

Sex-based differences in disease are a consequence of genetic differences that are attributable to X-chromosome inactivation, to differences in the expression of steroid hormones, and to differences in anatomy, gender or life experiences. The consequence of failing to include sex-based differences in study design and analyses has effectively led to 'one-drug' treatment regimens for both men and women. As a result of this bias, differences in drug efficacy and side-effect profiles reportedly led to the withdrawal of eight out of ten prescription drugs from the United States market in 2005, specifically owing to health issues in women ${ }^{5}$.

The under-representation of females in clinical studies has resulted in the disparity in both the understanding and the treatment of diseases in the individual sexes. Certainly, there are examples of specific clinical trials in which the proportion of male to female study participants reflects the prevalence of the condition under study in the general population. For example, more female than male participants have been enrolled in clinical trials for antidepressants and anti-inflammatory drugs, whereas primarily male participants (age $<60$ years) have been enrolled in clinical trials for drugs for cardiovascular diseases ${ }^{6,7}$. However, even in studies where female and male participation allow the analysis of the safety and efficacy of a therapy for both sexes or in the predisposed sex, inadequate emphasis has been placed on the identification of pharmacokinetic differences between males and females (BOX 1), or among females during different phases of their ovarian cycle.

The historical under-representation of women in clinical trails is understandable. In 1977, Food and Drug Administration (FDA) guidelines explicitly prohibited the participation of 'women of childbearing potential' in Phase 1 and early Phase 2 clinical trials. In 1993, the FDA made two changes in policy for the study and evaluation of drugs in women: first, sex-specific analyses of the safety and efficacy of the tested drugs were required; and second, it was no longer recommended that women of childbearing potential be restricted from participating in early drug trials. The National Institutes of Health (NIH) Revitalization Act of 1993 required that $\mathrm{NIH}$-funded clinical trials include women as subjects, and in 1998 the FDA mandated that new drug applications must include data on the safety and effectiveness of the drug for each sex. So far, however, there has been inadequate compliance to these policies. It is highly probable that the added economic cost of expanding a clinical trial to include

\section{Box 1 |Sex-based differences in pharmacokinetics and pharmacodynamics}

Pharmacokinetics describe the relationship between the dosage of a drug and its concentration over time in blood, plasma, cells and tissues. Factors that influence pharmacokinetics include bioavailability, distribution, metabolism and excretion. Sex-based differences in gastric emptying (which influences bioavailability), body composition — body weight, body fat, plasma volume, organ blood flow - (which influences distribution), hepatic enzymes that metabolize drugs (which influence drug metabolism) and renal clearance (which influences drug excretion), all contribute to sex-based differences in pharmacokinetics ${ }^{63}$. Pharmacodynamics relates pharmacokinetic parameters to pharmacological effect. Sex-based differences in pharmacodynamics are distinguished from sex-based differences in pharmacokinetics based on evidence that the same plasma concentration of a drug in males and females does not necessarily result in the same pharmacological outcome. 
appropriate numbers of women, including women during different hormonal phases, to afford sufficient power for the detection of sex-based differences both in pharmacokinetics and in clinical responses, is the main factor behind non-compliance.

In this Perspective, I review sex-based differences in the immune response in the context of autoimmune diseases and viral infections, and discuss the implications and clinical significance of these issues for drug development and effective health care.

\section{The $\mathrm{X}$ chromosome and disease}

The X chromosome encodes approximately 1,100 genes, most of which are distinct from the fewer than 100 genes that are expressed on the Y chromosome (FIG. 1). A priori, expression of the few unique Y-linked genes might underlie some sex differences in disease susceptibility. Notably, a recent study of transgenic mice that were created to compare disease susceptibility in XX and XY mice with a common gonadal type revealed that the XX-chromosome complement conferred a greater susceptibility to both SLE and experimental autoimmune encephalomyelitis (EAE), a mouse model of multiple sclerosis, compared with the XY-chromosome complement ${ }^{8}$. By contrast, normal males are more vulnerable to $\mathrm{X}$-linked diseases as they have a single X chromosome, whereas females have two, which provides the added biological advantage of the cellular mosaicism that is associated with X-inactivation ${ }^{9}$. Importantly, humans are an outbred species and therefore exhibit allelic diversity in $\mathrm{X}$-chromosome

\section{a Receptors \& associated proteins}

\begin{tabular}{|c|c|}
\hline AR & Androgen receptor \\
\hline AGTR2 & Angiotensin receptor 2 \\
\hline CSF2RA & $\begin{array}{l}\text { Colony-stimulating factor } 2 \text { receptor } \alpha \\
\text { (granulocyte-macrophage) }\end{array}$ \\
\hline GPCR & $\begin{array}{l}\text { G-protein coupled receptors } 23,50,101,112,119,174 \\
\text { and CX-chemokine receptor } 3\end{array}$ \\
\hline CYSLTR1 & Cysteinyl leukotriene receptor 1 \\
\hline IL-IRAPI & Interleukin-1 (IL-1) receptor accessory protein-like 1 \\
\hline IL-IRAP2 & IL-1 receptor accessory protein-like 2 \\
\hline IL-2RG & IL-2 receptor $\gamma$-chain \\
\hline IL-3RA & IL-3 receptor $\alpha$-chain \\
\hline IL-9R & IL-9 receptor \\
\hline IL-13RA1 & IL-13 receptor $\alpha$ l-chain \\
\hline IL-13RA2 & IL-13 receptor $\alpha 2$-chain \\
\hline IRAK & IL-1 receptor-associated kinase \\
\hline NGFRAPI & Nerve-growth-factor receptor associated protein 1 \\
\hline TLR7 & Toll-like receptor 7 \\
\hline TLR8 & Toll-like receptor 8 \\
\hline
\end{tabular}

\section{b Immune-response related proteins}

\begin{tabular}{ll} 
XSCID & X-linked severe combined immunodeficiency \\
ELK1 & Involved in B-cell development \\
\hline EPAG & Early lymphoid activation protein \\
GATA1 & GATA-binding protein 1 \\
GTD & Gonadotropin deficiency \\
IDDMX & X-linked susceptibility to insulin-dependent diabetes \\
IGBP1 & CD79A, immunoglobulin binding protein 1 \\
IGSF1 & Immunoglobulin superfamily member 1 \\
ITGB1BP2 & Integrin- $\beta$ 1-binding protein 2 \\
CD99 & Also known as MIC2; associated with T-cell function \\
MTCP1 & Mature T-cell proliferation 1 \\
PFC & Properdin P factor, complement \\
TIMP1 & Tissue inhibitor of metalloproteinase 1 \\
CD40L & CD40 ligand \\
Z39IG & An immunoglobulin superfamily protein
\end{tabular}

Figure 1 | Genes on the $\mathrm{X}$ chromosome with the potential to influence immunocompetence. Several proteins encoded by genes that are found on the $\mathrm{X}$ chromosome might underlie sex-based differences in immune responses. The proteins listed were selected from more than 1,100 identified genes on the $\mathrm{X}$ chromosome, and have been grouped according to their

\begin{tabular}{|c|c|}
\hline \multicolumn{2}{|c|}{ Transcriptional \& translational control effectors } \\
\hline RHOGAP & RAS homologue (RHO) GTPase activating proteins 4,6 \\
\hline CDC42GEF & Cell-division cycle 42 guanine-nucleotide-exchange factors 6,9 \\
\hline ETK & Also known as BMX \\
\hline BTK & Bruton agammaglobulinaemia tyrosine kinase \\
\hline CDX4 & Caudal homeobox transcription factor 4 \\
\hline TRAPI70 & A co-factor for SP1 transcription factor activation \\
\hline DUSP & Dual specificity phosphatases 9,21 \\
\hline EEF & Eukaryotic translation elongation factors $1 \alpha 13, \beta 4$ \\
\hline EIF & Eukaryotic translation initiation factor $1 \mathrm{~A}^{\star}, 2 \mathrm{a}$ \\
\hline FOXP3 & $\begin{array}{l}\text { Forkhead box P3 (associated with the development and } \\
\text { function of regulatory T cells) }\end{array}$ \\
\hline GAB3 & $\begin{array}{l}\text { Growth-factor-receptor-bound protein 2-associated binding } \\
\text { protein } 3\end{array}$ \\
\hline HDAC & Histone deacetylases 6,8 \\
\hline $\mathrm{IKK} \gamma$ & I $\kappa$ B kinase; also known as NEMO \\
\hline MAPKKK15 & Mitogen-activated protein kinase kinase kinase 15 \\
\hline NFKBRF & Nuclear factor- $\kappa \mathrm{B}(\mathrm{NF}-\kappa \mathrm{B})$ repressing factor \\
\hline NRK & NF- $\kappa B$-inducing kinase-related kinase \\
\hline NXF & Nuclear RNA export factors 2, 3, 4, 5 \\
\hline PAK3 & p21 (also known as CDKN1A)-activated kinase 3 \\
\hline PPP & Protein phosphatases $1,2^{\star}, 6$ \\
\hline PRKCl & Protein kinase $\mathrm{Ci}$ \\
\hline S6K & Ribosomal protein S6 kinase \\
\hline SWI/SNF & $\begin{array}{l}\text { SWI/SNF-related, matrix associated, actin-dependent regulator } \\
\text { of chromatin }\end{array}$ \\
\hline STK9 & Serine/threonine kinase 9 \\
\hline TAFI & TATA-box-binding protein-associated factor 1, TFIID subunit \\
\hline UBE1 & Ubiquitin-activating enzyme El \\
\hline UBE2A & Ubiquitin-conjugating enzyme E2A \\
\hline USP & Ubiquitin-specific proteases $9^{\star}, 11,26,27,511$ \\
\hline WASP & Wiskott-Aldrich syndrome protein \\
\hline
\end{tabular}

associated function as receptors and associated proteins (a), proteins related to the immune response (b) or proteins involved in transcriptional and translational control (c). Proteins with their definition and/or known function are listed. The proteins marked with an asterisk indicate those encoded by genes also found on the $Y$ chromosome. 
genes in contrast to inbred mice, in which maternal and paternal X-chromosome genes are identical. As a result, a mutation on the maternal X-chromosome gene might not exist in the paternal X-chromosome gene in humans.

$\mathrm{X}$-chromosome inactivation provides dosage compensation for X-linked genes between XX females and XY males. In a random process, one of the two $\mathrm{X}$ chromosomes is transcriptionally silenced early in female development, which leads to the mosaic expression of either the maternal $(\mathrm{Xm})$ or paternal $(\mathrm{Xp}) \mathrm{X}$ chromosome in different cell populations. Thus, as a result of X-inactivation, potentially half of the cells express an X-linked gene mutation in females, whereas all cells in males express an X-linked gene mutation. For example, a mutation in the $\gamma$-chain subunit that is common to receptors for interleukin 2 (IL-2), IL-4, IL-7, IL-9, IL-15 and IL-21, causes $\mathrm{X}$-linked severe combined immunodeficiency $(\mathrm{XSCID})^{10}$, a disease that was originally identified in a family of genetically related males ${ }^{11}$. In males, all immune-cell lineages that normally express this receptor subunit harbour the X-linked gene mutation, whereas $\mathrm{X}$-inactivation in females provides for restricted expression of the mutation and thereby diminishes the extent of immune deficiency.

IPEX (immune dysregulation, polyendocrinopathy, enteropathy, X-linked syndrome), a rare inflammatory disease that is caused by mutations in the gene that encodes the transcriptional regulator FOXP3 (forkhead box P3), destroys the immunoregulatory environment in affected male infants ${ }^{12}$. Without a functional FOXP3 protein, regulatory $\mathrm{T}\left(\mathrm{T}_{\mathrm{Reg}}\right)$ cells do not develop normally and the immune suppression that is mediated by this subset of T cells is absent. Again, X-inactivation gives females protection from IPEX; although 100\% of the cells in males that express FOXP3 display the mutation, the normal $\mathrm{T}_{\mathrm{Reg}}$ cells that express the wild-type gene are sufficient to maintain homeostasis in females.

In heterozygous females, metabolic cooperation can contribute an added advantage. More specifically, if the X-linked gene encodes a secreted protein, expression of the intact gene by some cells allows for the uptake of the protein by null cells that express the defective gene. For example, Hunter syndrome, a lysosomal storage disease that results in severe and progressive symptoms which affect many organs, is caused by defects in the enzyme iduronate sulphatose, which is synthesized in some cells of carrier females but not in others.

The defect in the mutant cells is corrected by the transfer of iduronate sulphatase from the wild-type cells that secrete the functional enzyme ${ }^{13}$. Accordingly, carrier females are usually unaffected by the X-linked iduronate sulphatase gene defect.

The skewing of $\mathrm{X}$-inactivation can provide another advantage to heterozygous females, as this process can lead to the selection of wild-type cells over mutant cells. For example, non-random inactivation of the $\mathrm{X}$ chromosome that carries the defective Wiskott-Aldrich syndrome gene (mutation of which causes a life-threatening immunodeficiency in affected males) occurs in the early-lineage haematopoietic cells of female carriers, thereby preventing the disease ${ }^{14}$. Although the skewing of X-inactivation to select for normal cells is beneficial and occurs in the majority of instances, extreme skewed X-inactivation has also been implicated in the aetiology of autoimmune diseases, such as scleroderma ${ }^{15}$. The skewed $\mathrm{X}$-inactivation of genes that are involved in antigen processing in discrete cell populations in the thymus, such as dendritic cells (DCs) that present antigen to T cells undergoing selection, might affect the recognition of self antigens and thereby cause a loss of immunological tolerance, which is a feature of many autoimmune diseases.

Viewed together, the genetic contribution of X-inactivation effectively provides females with a more extensive repertoire of proteins and the potential for diversity in many physiological processes, compared with males. However, the influence of X-inactivation and $\mathrm{X}$-linked gene mutations to disease susceptibility is complicated by other factors. More specifically, mutations in non-X-linked genes that are associated with chromatin remodelling or transcriptional and translational events also have the potential to influence $\mathrm{X}$-inactivation and the expression of genes from the X chromosome. Moreover, mutations in any of the transcriptional and translational genes that are encoded on the $\mathrm{X}$ chromosome (FIG. 1) could influence gene expression from other chromosomes.

\section{Sex-based differences in immunity Oestrogen influences immunocompetence.} As a general rule, females exhibit more robust cell-mediated and humoral immune responses to antigenic challenges, such as infection and vaccination, compared with males. Oestrogens, such as $17 \beta$-oestradiol (also known as E2) and oestriol, progesterone and testosterone, can mediate many of the sex-based differences in immune responses ${ }^{16}$. Oestrogens exert their effects by binding to cognate intracellular receptors. The two subtypes of the receptor for oestrogens, oestrogen receptor $\alpha$ (ER $\alpha$; also known as ESR1) and ER $\underline{\text { Elso known }}$ as ESR2), are expressed by many types of immune cell, including T cells, B cells, dendritic cells (DCs), macrophages, neutrophils and natural killer (NK) cells, which suggests that oestrogens might have a role in the regulation of immunocompetence (FIG. 2). Oestrogen-ER $\alpha$ or $-\mathrm{ER} \beta$ complexes translocate to the nucleus, where they bind to distinct ER-responsive elements in the promoters of target genes, thereby regulating transcriptional activity. In addition, oestrogen-ER $\alpha$ or oestrogen-ER $\beta$ complexes might regulate the transcriptional activity of genes that do not have an ER-responsive element by recruiting co-regulatory proteins that can activate or repress transcription ${ }^{17}$. Interestingly, ligands for $E R \alpha$ and $E R \beta$ exert different influences on the course of EAE. More specifically, treatment with either an ER $\alpha$ ligand or with $17 \beta$-oestradiol (which binds to both $\mathrm{ER} \alpha$ and $\operatorname{ER} \beta$ ) is anti-inflammatory and abrogates the onset of EAE, whereas treatment with a specific ER $\beta$ ligand is neuroprotective but not anti-inflammatory ${ }^{18}$.

Women have higher $\mathrm{CD}^{+} \mathrm{T}$-cell numbers than men $^{19}$, and $\mathrm{T}_{\mathrm{Reg}}$-cell frequencies within the $\mathrm{CD} 4^{+}$population undergo profound changes during the ovarian cycle that potentially affect immunoregulation. $\mathrm{T}_{\text {Reg }}$-cell numbers increase during the follicular phase of the menstrual cycle, when oestrogen levels are high, and decrease during the luteal phase, when oestrogen levels are low $^{20}$ (FIG. 3). $\mathrm{T}_{\text {Reg }}$ cells regulate the size of the peripheral T-cell pool, modulate immune responses to infection and participate in the maintenance of self-tolerance by suppressing immune responses mediated by autoreactive $\mathrm{T}$ cells that can contribute to autoimmune disease ${ }^{21}$. Accordingly, variation in the number of $\mathrm{T}_{\mathrm{Reg}}$ cells over the course of the menstrual cycle will influence immune responses. In addition, $\mathrm{T}_{\mathrm{Reg}}$-cell functional deficits have been implicated in autoimmune diseases, such as multiple sclerosis and rheumatoid arthritis ${ }^{22,23}$.

Distinct from the effects on $\mathrm{T}_{\mathrm{Reg}}$-cell numbers, oestrogens affect the expression of some chemokine receptors by $\mathrm{T}$ cells. Oestrogens selectively increase the expression and responsiveness of CC-chemokine receptor 5 (CCR5) and CCR1 in $\mathrm{CD}^{+}$ T cells, which has important implications for T-cell homing in the context of both infection and autoimmunity ${ }^{24}$. 


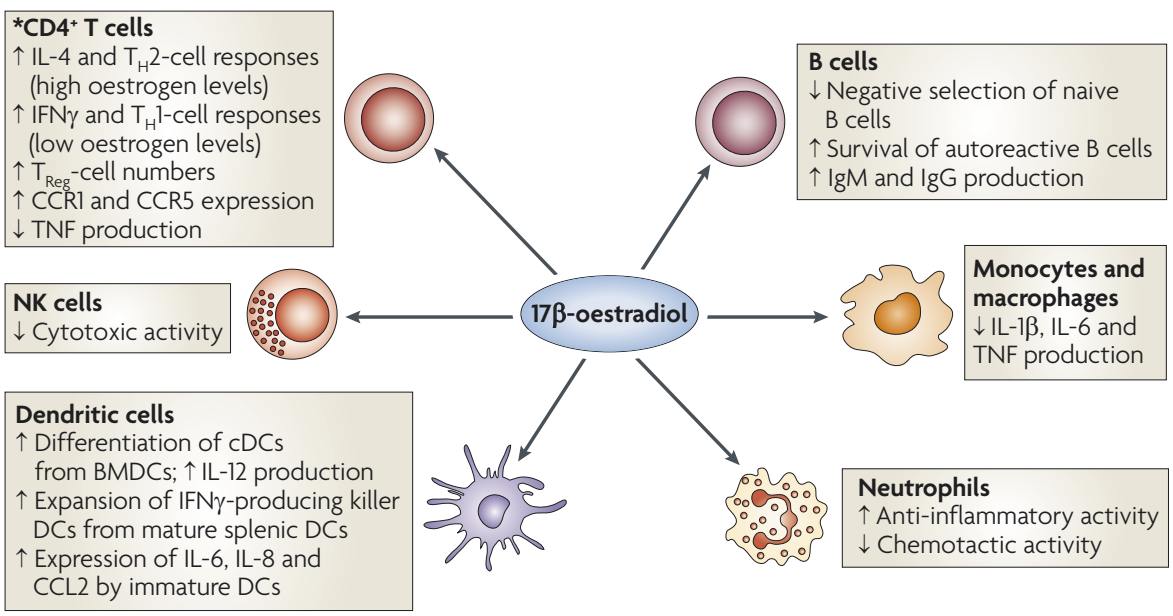

Figure 2 | The effects of $\mathbf{1 7} \beta$-oestradiol on immunocompetence. The activation of oestrogen receptors expressed by T cells, B cells, dendritic cells (DCs), macrophages, neutrophils and natural killer (NK) cells influences immunocompetence. Oestrogens, such as $17 \beta$-oestradiol, exert a biphasic effect on $T$ helper $\left(T_{H}\right)$-cell polarization: low levels promote $T_{H} 1$-cell differentiation and higher levels promote $\mathrm{T}_{\mathrm{H}} 2$-cell polarization, with consequent effects on the production of cytokines that are associated with each of the $\mathrm{T}_{\mathrm{H}}$-cell populations. Oestrogen increases the size of the $\mathrm{T}_{\text {Reg }}$-cell population. In addition, oestrogen increases CC-chemokine receptor 1 (CCR1) and CCR5 expression and decreases tumour-necrosis factor (TNF) production by $C D 4^{+} T$ cells. Oestrogen decreases the negative selection of naive $B$ cells, enhances the survival of autoreactive B cells and enhances polyclonal B-cell activation and immunoglobulin production. The inhibition of CD16 expression by oestrogen in monocytes and macrophages leads to the reduced production of the pro-inflammatory cytokines interleukin-1 (IL-1 $\beta$ ), IL-6 and TNF. In neutrophils, oestrogen upregulates the production of nitric-oxide synthase and nitric oxide, thereby promoting their anti-inflammatory effects, and decreases their chemotactic activity. Oestrogen promotes the differentiation of conventional DCs (cDCs) from bone-marrow-derived DC precursors (BMDCs), and increases their IL-12 production, whereas exposure of mature splenic DCs to oestrogen results in the expansion of interferon- $\gamma$ (IFN $\gamma$ )-producing killer DCs. $17 \beta$-oestradiol treatment increases the secretion of IL-6, IL-8 and CC-chemokine ligand 2 (CCL2) by immature DCs (iDCs). Oestrogen also reduces the cytotoxicity of NK cells. * refer to FIG. 3.

Oestrogens might also exert a biphasic effect on Thelper $1\left(\mathrm{~T}_{\mathrm{H}} 1\right)$-cell versus $\mathrm{T}_{\mathrm{H}}$ 2-cell differentiation. Specifically, low doses of oestrogens have been associated with $\mathrm{T}_{\mathrm{H}} 1$-cell responses and enhanced cellmediated immunity, whereas high doses of oestrogens promote $\mathrm{T}_{\mathrm{H}}$ 2-cell and humoral responses. Interestingly, there is evidence to suggest that the contrasting effects of oestrogens on T-bet, a master regulator of $\mathrm{T}_{\mathrm{H}} 1$-cell differentiation, and interferon regulatory factor-1 (IRF1), a transcription factor that is associated with the regulation of interferon- $\gamma$ (IFN $\gamma$ ), may be dose-dependent and account for $\mathrm{T}_{\mathrm{H}} 1$-cell versus $T_{H} 2$-cell differentiation. Lower levels of oestrogen might contribute to increased expression of T-bet, whereas higher levels might downregulate the expression of IRF1 (REF. 25). Although low doses of oestrogen are associated with enhanced IFN $\gamma$ expression by $\mathrm{T}_{\mathrm{H}} 1$ cells, higher doses of oestrogen enhance IL-4 production. In addition, oestrogens are negative regulators of $\mathrm{CD} 4^{+}$ T-cell-derived tumour-necrosis factor (TNF) (FIG. 2).
The influence of oestrogens on the $\mathrm{T}_{\mathrm{H}}$-cell bias is exemplified by the hormonal environment during pregnancy, in which increased oestrogen and progesterone levels in the third trimester favour the generation of $\mathrm{T}_{\mathrm{H}} 2$-cell responses. Notably, oestriol, an oestrogen metabolite that is derived from the placenta, is only produced in significant amounts during pregnancy. For some autoimmune diseases, hormonal fluctuations during pregnancy affect disease activity: the $\mathrm{T}_{\mathrm{H}}$ 2-cell-type environment contributes to enhanced antibody production that exacerbates $\mathrm{SLE}^{25}$, whereas suppression of $\mathrm{T}_{\mathrm{H}} 1$-cell responses by oestrogens results in decreased disease activity in patients with rheumatoid arthritis ${ }^{26}$. For multiple sclerosis, recent evidence from EAE models suggests that the decrease in disease activity during pregnancy is probably associated with an immunoregulatory environment rather than with the suppression of $\mathrm{T}_{\mathrm{H}} 1$-cell responses ${ }^{27}$. These findings have led to the initiation of several pilot clinical studies to examine the effects of oestriol therapy in multiple sclero$\mathrm{sis}^{28}$. Notably, the remission of rheumatoid arthritis and multiple sclerosis during pregnancy is often followed by a flare of disease activity post-partum, when oestrogen and progesterone levels fall ${ }^{29,30}$.

Oestrogens also affect B-cell development by decreasing negative selection of naive immature B cells, enhancing the survival of autoreactive $B$ cells ${ }^{31}$ and enhancing polyclonal activation of $\mathrm{B}$ cells, which leads to higher serum levels of IgG and IgM (mediated by CD95-CD95L interactions ${ }^{32}$ ). These B-cell effects might contribute to the increased incidence of many autoimmune diseases in women.

The effects of oestrogens on the innate immune responses that are mediated by monocytes and macrophages are largely repressive $^{33}$. CD16 (also known as FcyRIIIA) is a receptor expressed by monocytes and macrophages that is activated by self antigens and rheumatoid factor to induce signalling cascades that stimulate cytokine production. Oestrogens can modulate CD16 expression by monocytes and macrophages. The interaction of ER $\alpha$ with the CD16 promoter suppresses the expression of CD16 (REF. 34) and, consequently, oestrogens reduce monocyte and macrophage production of the proinflammatory cytokines IL- $1 \beta$, IL- 6 and TNF in vitro ${ }^{35}$. In addition, oestrogens decrease plasma IL-6 levels, and the production of TNF and IL- $1 \beta$ is increased during the luteal phase (low oestrogen) compared with the follicular (high oestrogen) phase of the ovarian cycle ${ }^{16}$.

In addition to their anti-inflammatory effects on monocytes that are mediated by decreasing the production of proinflammatory cytokines, oestrogens exert anti-inflammatory effects on neutrophils by increasing nitric-oxide synthase expression and nitric-oxide production ${ }^{36}$. Additionally, progesterone enhances the chemotactic activity of neutrophils, whereas oestrogens decrease $\mathrm{it}^{37}$.

Oestrogens also have an effect on other innate immune cells. More specifically, oestrogens decrease the cytotoxicity of NK cells ${ }^{38}$. In addition, recent data indicate that oestrogens can also regulate DC development ${ }^{39}$; exposure of bone-marrow DC precursors to oestrogens facilitated their development into conventional DCs that secreted pro-inflammatory IL-12. Furthermore, exposure of mature splenic DCs to oestrogens resulted in the expansion of IFN $\gamma$-producing killer DCs (which had a CD $11 \mathrm{c}^{+} \mathrm{MHC}$ class $\mathrm{II}^{+} \mathrm{CD} 49 \mathrm{~b}^{+} \mathrm{NK} 1.1^{\text {high }}$ phenotype $)^{39}$. $17 \beta$-oestradiol treatment of immature DCs increases IL-6, IL-8 (also known as CXCL8) and CCL2 (also known as MCP1) production ${ }^{40}$ (FIG. 2). 

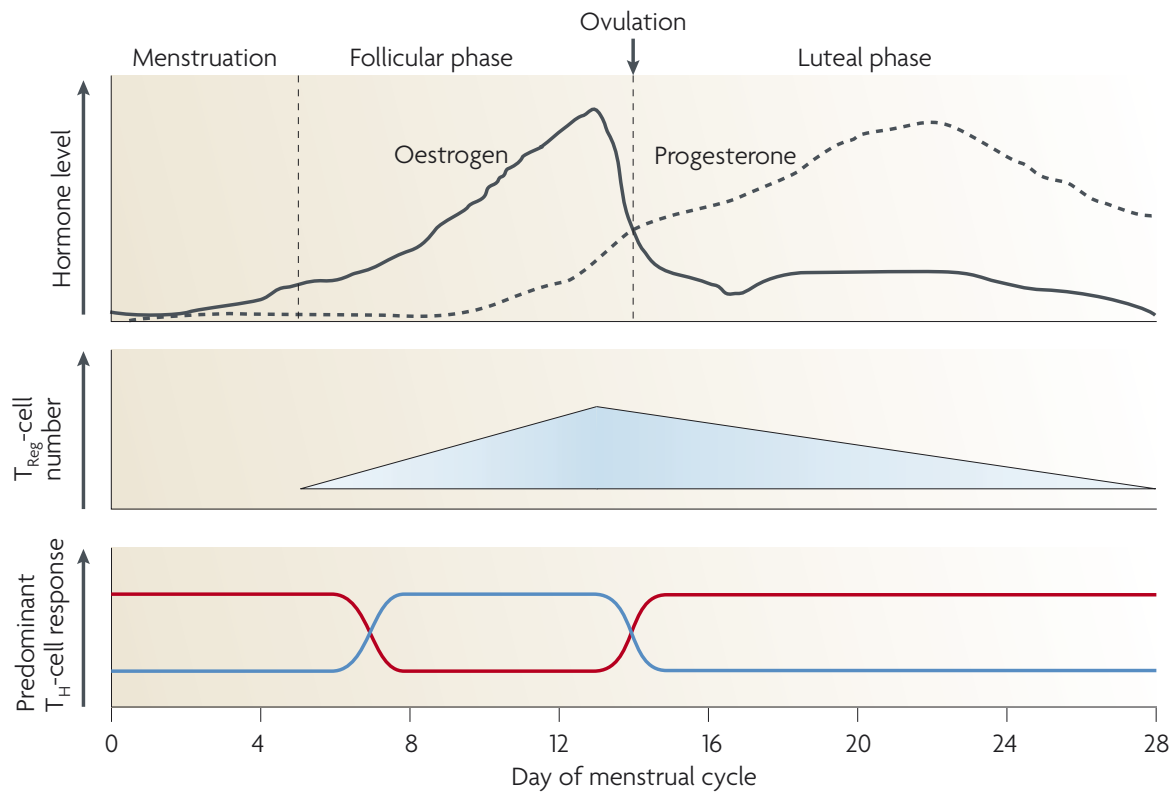

$\mathrm{T}_{\mathrm{H}}$-cell response $\quad \mathrm{T}_{\mathrm{H}} 2$-cell response

Figure 3 | Oestrogen and progesterone effects on T-cell responses during the menstrual cycle. Variations in oestrogen and progesterone levels during the different phases of the menstrual cycle influence T helper $1\left(\mathrm{~T}_{\mathrm{H}} 1\right)-, \mathrm{T}_{\mathrm{H}} 2$ - and T regulatory $\left(\mathrm{T}_{\mathrm{Reg}}\right)$-cell populations. The upper panel illustrates fluctuations in the levels of oestrogen and progesterone during the different phases of the 28-day menstrual cycle. In the middle and lower panels, the corresponding changes in the size of the $T_{\text {Reg }}$-cell population and the $T_{H}$-cell bias, respectively, are shown.

Moreover, oestrogens can further shape the immune response by affecting microvascular endothelial cells, which actively recruit immune and inflammatory cells to lymphoid and peripheral tissues through the expression of adhesion molecules and chemokines $^{41}$. More specifically, oestrogens influence endothelial-cell function by differentially regulating ER $\alpha$ and ER $\beta$ expression and by selectively enhancing the expression of adhesion molecules and chemokines.

Notably, the effects of androgens, such as testosterone, on immune function are largely suppressive, thereby leading to decreased T- and B-cell proliferation and decreased immunoglobulin and cytokine production $^{42}$. Testosterone decreases the expression of macrophage and monocyte Toll-like receptor 4 (TLR4), a receptor that is involved in activating the innate immune system in response to pathogen challenge, and this decrease in TLR4 expression provides a potential underlying mechanism for the immunosuppressive effects of testosterone $\mathrm{e}^{43}$. It is also worth noting that oestrogens can be derived from the conversion of androgens by aromatase enzymes. The presence of the pro-inflammatory cytokines TNF, IL- $1 \beta$ and IL- 6 in the affected tissues of individuals with rheumatoid arthritis ${ }^{44}$ and $\mathrm{SLE}^{45}$ has been associated with increased aromatase activity and a corresponding acceleration in the synthesis of oestrogens. The paracrine effects of increased oestrogen levels in affected tissues could thereby influence disease activity by affecting the function of resident T cells, B cells, monocytes and macrophages, as described previously. However, the role of oestrogen metabolites, such as $16 \alpha$-hydroxyestrone and 2-hydroxyoestrogen, as pro- and anti-inflammatory mediators, respectively, in these chronic inflammatory diseases remains unclear ${ }^{42}$.

Clearly, the widespread effects of oestrogens and progesterone on different immune cells have implications on disease susceptibility for both sexes. However, although it is known that sex differences predispose susceptibility to many autoimmune diseases, distinguishing sex-specific effects of sex steroids in pathology and therapy has received limited attention. A notable exception is in patients with multiple sclerosis; testosterone treatment exhibited potential neuroprotective effects in men with relapsing-remitting multiple sclerosis ${ }^{46}$. Given the evidence of disease remission during pregnancy, and the potential neuroprotective effects of ER ligands ${ }^{18}, 17 \beta$-oestradiol treatment strategies offer the prospect of a novel therapy for women with multiple sclerosis.
The effects of oestrogens and hormone fluctuations that are related to the reproductive phase on immune-cell development and function, on cytokine and chemokine production, and on the migration of cells to sites of inflammation, all influence immunocompetence. Accordingly, hormones can affect the pathogenesis of chronic autoimmune and inflammatory diseases and can contribute to the higher incidence of certain autoimmune diseases in women. In addition to influencing the incidence and progression of certain autoimmune diseases, sex-based differences that affect immunocompetence can also influence susceptibility to infections and the severity of subsequent illnesses.

Sex-based differences in infectious diseases. Although the prevailing dogma is that females typically mount more robust immune responses to viral infections than males ${ }^{1}$, changes in hormone concentration owing to the menstrual cycle, contraception usage, hormone-replacement therapy and pregnancy can all influence the immune response to pathogens. In addition, men and women are also differentially susceptible to several DNA and RNA viruses, such as HSV-2, HIV, measles virus, hantaviruses and vesicular stomatitis virus (VSV) ${ }^{1}$. However, close scrutiny of the scientific literature reveals a scarcity of publications that have examined sex-based differences in human viral infectious diseases, as most studies have been carried out in mice or rats.

Data from rodent studies do, however, provide some insights into the mechanisms that contribute to sex-based differences in disease severity. Coxsackievirus B3 (CVB3) infection is associated with myocarditis ${ }^{47}$, and although men and women are equally susceptible to CVB3 infection, the cardioprotective effects of oestrogens have been linked to a lower incidence of heart disease in women ${ }^{48}$. Accumulating data indicate that the differences in the severity of myocarditis between the sexes are not limited to differences in the levels of viral replication in the heart, but are attributable to differences in the immune response to infection ${ }^{49,50}$. The higher levels of oestrogens in women decrease the expression of pro-inflammatory cytokines, whereas the more robust proinflammatory immune response to CVB3 infection in males increases inflammation in the heart. Recent data from mouse studies suggest that oestrogens mediate a decrease in the numbers of mast cells and macrophages in the infected hearts of females, with associated upregulation of T-cell immunoglobulin domain and mucin domain-3 (TIM3; also 
known as HAVCR2) by these cells. In turn, TIM3 inhibits TLR4 expression, thereby regulating the inflammatory response, and increases the size of the $\mathrm{T}_{\mathrm{Reg}}$-cell population. By contrast, infected male hearts show increased numbers of mast cells and macrophages with high expression of TLR4. This TLR4 expression in males inhibits downregulation of the inflammatory response and decreases TIM3 expression, thereby reducing the size of the $\mathrm{T}_{\text {Reg }}$-cell population and inhibiting TIM3-mediated apoptosis ${ }^{50}$.

Similar to CVB3 infection in humans, male and female rats are equally susceptible to infection with the hantavirus Seoul virus, but females mount a more robust immune response to infection that results in decreased viral shedding and a lower viral load in target organs ${ }^{51}$. An analysis of the lungs of hantavirus-infected female rats revealed a pattern of gene expression that is associated with a robust innate antiviral immune response, namely increased levels of genes that encode microbial pattern recognition receptors, IFN $\beta$ and IFN-inducible signalling effectors, compared with the lungs of infected male rats ${ }^{52}$. Human studies indicate that more males than females are infected with hantaviruses ${ }^{53}$, which perhaps indicates that the more rigorous innate immune response that was identified in infected female rats also occurs in humans. Moreover, hantavirusinfected females had significantly higher plasma levels of IL-9 and lower levels of the pro-inflammatory chemokines CXCL8 and CXCL10 than males, which further supports the existence of sex-dependent differences in immune responses to viral infections ${ }^{54}$. Studies in mice suggest that sex-based differences in the susceptibility to viral infections of the central nervous system (CNS), such as VSV, also correspond to enhanced immunity in the CNS in females and are associated with recovery from this neurotropic viral infection ${ }^{55}$. Finally, although age was an important predictor of mortality from severe acute respiratory syndrome (SARS) during the virus outbreak in 2003, females had lower mortality rates than males, even after adjusting for age. It is tempting to speculate that a more robust innate antiviral immune response in females might have accounted for this reduced mortality rate r. $^{56}$.

By contrast, Dengue virus infection is more severe in female children than in male children $^{57}$. One possible explanation for this is that the stronger humoral immune response to the virus in females might be linked to the development of crossreactive, non-neutralizing antibodies that allow the virus replication to proceed unchecked.
However, for herpesvirus infections, the stronger humoral immune response that is exhibited by females is advantageous, as men are more likely than women to be seronegative for the gammaherpesvirus Epstein-Barr virus (EBV), and seropositive women have higher EBV-specific antibody titres than seropositive men $^{58}$. In addition, women infected with cytomegalovirus (CMV) produce higher levels of IFN $\gamma$ and IL-2 than CMV-infected men, which is consistent with the more robust cell-mediated immune responses found in women ${ }^{59}$. These findings might help to explain why only females developed protective immunity to viral antigens in a Phase III HSV-2 clinical vaccine trial ${ }^{60}$ and further emphasize the importance of evaluating responses of both sexes in such studies.

The World Health Organization (WHO) estimates that one million new cases of STIs occur daily worldwide. For both HIV and HSV-2 (the most common sexually transmitted virus), the rate of transmission from males to females is greater than from females to males, which contributes to the higher prevalence of STIs in females. The susceptibility of the mucosal surface of the female genital tract to infection with both HIV and HSV-2 is influenced by oestrogen and progesterone levels. Specifically, progesterone is protective and decreases viral shedding, whereas oestrogens increase viral shedding $^{61,62}$. In the context of HIV infection, levels of viral RNA in the plasma are consistently lower in women than men. Given that the initial viral load after seroconversion is predictive of the likelihood of progression to AIDS in men, and that women have higher $\mathrm{CD}^{+}$T-cell counts, one would anticipate that women would therefore be at a lower risk of AIDS; however, this is not the case ${ }^{63}$.

Collectively, these findings indicate that sex-dependent factors influence both the susceptibility to numerous viral infections and their progression. For several viral infections, susceptibility is identical among men and women. However, fluctuations in oestrogen and progesterone levels directly affect immune responses at mucosal tissues, thereby influencing female susceptibility to sexually transmitted viral infections. The effects of oestrogens on cytokine and chemokine production promote an anti-inflammatory environment, which offers females protection from the immunopathology that is associated with certain viral infections. Notably, the changes in oestrogen levels in women that are determined by ovarian activity directly influence the profile of the immune response; low oestrogen levels are associated with a $\mathrm{T}_{\mathrm{H}} 1$-cell polarized response, which is linked to the production of cytokines with antiviral activity, whereas high oestrogen levels are associated with $\mathrm{T}_{\mathrm{H}} 2$-cell polarization and the production of cytokines that promote humoral immunity. The more vigorous humoral immune response in females is also a consequence of the direct effects of oestrogens on B-cell function, which enhances neutralizing antibody production and thereby assists in viral clearance.

\section{The impact of gender differences. Differences} in many infectious-disease processes between men and women arise based on sex and gender. Sex refers to the biological differences between males and females (as described previously in this Perspective article), whereas gender refers to the differences between males and females that are determined by cultural and societal factors.

A recent joint venture publication of the Departments of Gender, Women and Health, and Epidemic and Pandemic Alert and Response at the WHO noted that the reporting of and responses related to infectious outbreaks rarely included data on gender or sex differences ${ }^{56}$. In 2001, The Institute of Medicine suggested that assessments of differences in the incidence of many infectious diseases between males and females should also take into account differences in disease exposure ${ }^{64}$.

Perhaps the most compelling example in which gender is a significant factor in disease susceptibility relates to the contextual cultural and social realities that put women at high risk for contracting HIV in sub-Saharan Africa. Another interesting example is that of measles, where infection contracted from a child of the opposite sex is more severe than infection contracted from a child of the same sex ${ }^{64}$. Moreover, secondary cases of measles in the home are more severe and have higher mortality rates. The severity of secondary cases may relate to higher virus absorption that is associated with a higher infective dose ${ }^{65}$. In certain societies, fatality rates of measles cases are higher for females than for males, as girls remain at home and are therefore at a higher risk of infection from siblings inside the home. In addition, males can contract the disease outside the home and pass on the secondary, more severe infection to the females who remain inside the home ${ }^{62}$. Greater severity has also been associated with the transmission between the sexes of Varicella-Zoster virus (which causes chickenpox) and polio virus ${ }^{64}$. Thus, the increased severity of some infectious diseases in females can be the result of gender rather than sex differences. 


\section{Clinical implications}

The basis for new therapeutic strategies and improved disease management includes learning more information about hostpathogen interactions and increasing our knowledge regarding the aetiology and pathogenesis of individual autoimmune diseases. The preceding discussion illustrates how sex-based differences in immune responses contribute to disease susceptibility and severity, which are regulated in part by interactions between sex steroid hormones and the immune system. In addition, a role for the sex-chromosome complement in determining the female bias of autoimmune diseases has been recognized ${ }^{8}$. Interestingly, there is evidence that the male sex-chromosome complement and male sex hormones might, under certain circumstances, have compensatory effects on the immune response and might thereby decrease the differences in the immune response between males and females that are conferred by sex chromosomes alone ${ }^{66}$. Specifically, compensatory effects of sex chromosome complement and sex hormones on an autoantigen-specific immune response were observed in a study of mice deficient in the testis-determinant Sry gene, which allowed the production of mice that differ in sex-chromosome complement while having the same gonadal type, and which used gonadectomization to address sex hormone influences ${ }^{66}$.

With few exceptions, however, sexdependent differences in immune responses to infectious pathogens have been disregarded, and most studies of autoimmunity have not accounted for sex-related influences. In addition, studies of inbred mice cannot address the contributions of allelic diversity of genes on the $\mathrm{X}$ chromosome between males and females, and as such, investigation of sex-based differences in immune responses using mouse models are flawed. Therefore, accurate analyses of sexbased differences in immune responses will require examination of human tissues.

So far, drug development has proceeded without the consideration of sex-based differences in disease susceptibility or severity, which is particularly unfortunate as identification of sex-dependent factors could reveal potential new therapeutic targets. Moreover, drug development has so far not adequately addressed the accumulating evidence that suggests that sex-based differences affect pharmacokinetics and pharmacodynamics (BOX 1). Antiretroviral therapies for HIV exemplify this, as various studies of these drugs have shown that women have more frequent and serious adverse events than men $^{67}$, which is probably a consequence of sex-based differences in the pharmacokinetics and pharmacodynamics of antiretroviral drugs $^{68}$. Overall, women are at a greater risk of suffering from adverse drug reactions from medications that are already on the market as a result of their under-representation in clinical trials and owing to the lack of detailed pharmacokinetic and pharmacodynamic studies that are sufficiently powered to detect sex-based effects. Published data on drug efficacy segregated by sex is also lacking. For example, data regarding sex-based differences in viroimmunologic outcomes in HIVinfected men and women on antiretroviral therapy are inconclusive, largely because these studies are underpowered to detect these differences ${ }^{69-71}$.

Together, these data indicate that the consequences of not considering sex-based differences in human illnesses are significant and unacceptable. With the advent of human tissue biobanks, there is the opportunity to investigate human tissues ex vivo to examine sex-based differences in immune responses. Disease-specific tissue biobanks in which individual patients provide multiple specimens over the course of disease offer the added advantage of allowing for the analysis of sex-dependent differences in the context of hormonal fluctuations in females. Prospective banking therefore requires that data on the phase of the menstrual cycle, pregnancy, contraception use and hormonereplacement therapy must be acquired when tissue specimens are collected from females. Future studies must address sexbased differences in immune responses to infectious and autoimmune diseases in all aspects of scientific inquiry and make these considerations a priority for comprehensive and effective health care. The responsibility resides with health-research funding agencies, including federal government drug-licensing agencies, to insist that research investigations are stratified to include sex-dependent analyses.

Eleanor N. Fish is at the Toronto General Research Institute, University Health Network, Department of Immunology, University of Toronto, Women's College Research Institute, 67, College Street, Toronto Ontario M5G 2M1, Canada. e-mail:en.fish@utoronto.ca doi: 10.1038/nri2394

1. Klein, S. L. The effects of hormones on sex difference in infection: from genes to behavior. Neurosci. Biobehav. Rev. 24, 627-638 (2000)

2. Roberts, C. W., Walker, W. \& Alexander, J. Sexassociated hormones and immunity to protozoan parasites. Clin. Microbiol. Rev. 14, 476-488 (2001). Lockshin, M. D. Sex differences in autoimmune disease. Lupus 15, 753-756 (2006).

4. Whitacre, C. C. Sex differences in autoimmune disease Nature Immunol. 2, 777-780 (2001).
5. Simon, V. Wanted: women in clinical trials. Science 308, 1517 (2005)

6. Bartlett, C. et al. The causes and effects of sociodemographic exclusions from clinical trials. Health Technol. Assess. 9, 1-171 (2005).

7. Mullner, M., Vamvakas, S., Rietschel \& van ZwietenBoot, B. J. Are women appropriately represented and assessed in clinical trials submitted for marketing authorization? A review of the database of the European Medicines Agency. Int. J. Clin. Pharmacol. Ther. 45, 477-484 (2007).

8. Smith-Bouvier, D. L. et al. A role for sex chromosome complement in the female bias in autoimmune disease. J. Exp. Med. 205, 1099-1108 (2008)

9. Migeon, B. R. The role of $X$ inactivation and cellular mosaicism in women's health and sex-specific diseases. JAMA 295, 1428-1433 (2006).

10. Schmalstieg, F. C. \& Goldman, A. S. Immune consequences of mutations in the human common $\gamma$-chain gene. Mol. Genetics Metabol. 76, 163-171 (2002).

11. Brooks, E. G. et al. A novel X-linked combined immunodeficiency disease. J. Clin. Invest. $\mathbf{8 6}$ 1623-1631 (1990).

12. van der Vliet. H. J. \& Nieuwenhuis, E. E. IPEX as a result of mutations in FOXP3. Clin. Dev. Immunol. 2007, 1-5 (2007).

13. Fratantoni, J. C., Hall, C. W. \& Neufeld, E. F. Hunter and Hurler syndromes: mutual correction of the defect in cultured fibroblasts. Science 162, 570-572 (1968).

14. Wengler, G., Gorlin, J. B., Williamson, J. M., Rosen, F. S. \& Bing, D. H. Nonrandom inactivation of the $X$-chromosome in early lineage hematopoietic cells in carriers of Wiskott-Aldrich syndrome. Blood 85 , 2471-2477 (1995).

15. Ozbalkan, $Z$ et al. Skewed $X$ chromosome inactivation in blood cells of women with scleroderma. Arthritis Rheum. 52, 1564-1570 (2005).

16. Bouman, A., Heineman, M. J. \& Faas, M. M. Sex hormones and the immune response in humans. Hum. Reprod. Update 11, 411-423 (2005).

17. McDonnell, D. P. \& Norris, J. D. Connections and regulation of the human estrogen receptor. Science 296, 1642-1644 (2002)

18. Tiwari-Woodruff, S., Morales, L. B. J., Lee, R. $\delta$ Voskuhl, R. R. Differential neuroprotective and anti inflammatory effects of estrogen receptor (ER) $\alpha$ and ER $\beta$ ligand treatment. Proc. Natl Acad. Sci. USA 104 14813-14818 (2007).

19. Amadori, A. et al. Genetic control of the CD4/CD8 T cell ratio in humans. Nature Med. 1, 1279-1283 (1995).

20. Arruvito, L., Sanz, M., Banham, A. H. \& Fainboim, L. Expansion of $\mathrm{CD}^{+}{ }^{+} \mathrm{CD} 25^{+}$and FOXP3 + regulatory $T$ cells during the follicular phase of the menstrual cycle: implications for human reproduction. J. Immunol. 178, 2572-2578 (2007)

21. Tang, Q. \& Bluestone, J. A. The Foxp3+ regulatory T cell: a jack of all trades, master of regulation. Nature Immunol. 9, 239-244 (2008).

22. Viglietta, V., Baecher-Allan, C., Weiner, H. L. \& Haefler, D. A. Loss of functional suppression by $\mathrm{CD} 4{ }^{+} \mathrm{CD} 25^{+}$regulatory $\mathrm{T}$ cells in patients with multiple sclerosis. J. Exp. Med. 199, 971-979 (2004).

23. Lawson, C. A. et al. Early rheumatoid arthritis is associated with a deficit in the $\mathrm{CD} 4{ }^{+} \mathrm{CD} 25^{\text {high }}$ regulatory $\mathrm{T}$ cell population in peripheral blood. Rheumatology 45, 1210-1217 (2006).

24. Mo, R. et al. Estrogen regulates CCR gene expression and function in T lymphocytes. J. Immunol. 174, 6023-6029 (2005).

25. Pernis, A. B. Estrogen and $\mathrm{CD} 4^{+} \mathrm{T}$ cells. Curr. Opin Rheumatol. 19, 414-420 (2007).

26. Nelson, J. L. \& Ostensen, M. Pregnancy and rheumatoid arthritis. Rheum. Dis. Clin. North Am. 23, 195-212 (1997).

27. McClain, M. A. et al. Pregnancy suppresses experimental autoimmune encephalomyelitis through immunoregulatory cytokine production. J. Immunol. 179, 8146-8152 (2007).

28. Antonio, M., Patrizia, F., Itaria, I. \& Paolo, F. A rationa approach on the use of sex steroids in multiple sclerosis. Recent Patents CNS Drug Discov. 3, 34-39 (2008).

29. Olsen, N. J. \& Kovacs, W. J. Hormones, pregnancy, and rheumatoid arthritis. J. Gend. Specif. Med. 5, 28-37 (2002)

30. Houtchens, M. K. Pregnancy and multiple sclerosis. Semin. Neurol. 27, 434-441 (2007). 
31. Grimaldi, C. M. Cleary, J., Dsagtas, A. S., Moussai, D. \& Diamond, B. Estrogen alters thresholds for B cells apoptosis and activation. J. Clin. Invest. 109 1625-1633 (2002).

32. Lamason, R. et al. Sexual dimorphism in immune response genes as a function of puberty. $B M C$ Immunol. 7, 1-14 (2006).

33. Harkonen, P. L. \& Vaananen, H. K. Monocytemacrophage system as a target for estrogen and selective estrogen receptor modulators. Ann. N. Y. Acad. Sci. 1089, 218-227 (2006).

34. Kramer, P. R., Winger, V. \& Kramer, S. F. 17 $\beta$-estradio utilizes the estrogen receptor to regulate CD 16 expression in monocytes. Mol. Cell. Endocrinol. 279, 16-25 (2007).

35. Kramer, P. R Kramer, S. F \& Guan, G. 17ß-estradiol regulates cytokine release through modulation of CD 16 expression in monocytes and monocyte-derived macrophages. Arthritis Rheum. 50, 1967-1975 (2004).

36. Garcia-Duran, M. et al. Estrogen stimulates neuronal nitric oxide synthase protein expression in human neutrophils. Circ. Res. 85, 1020-1026 (1999).

37. Miyagi, M., Aoyama, H., Morishita, M. \& Iwamoto, Y. Effects of sex hormones on chemotaxis of human peripheral polymorphonuclear leukocytes and monocytes. J. Periodontol. 63, 28-32 (1992).

38. Hao, S. et al. Modulation of $17 \beta$-estradiol on the number and cytotoxicity of NK cells in vivo related to $\mathrm{MCM}$ and activating receptors. Int. Immunopharmacol. 7, 1765-1775 (2007).

39. Siracusa, M. C., Overstreet, M. G., Housseau, F., Scott, A. L. \& Klein, S. L. $17 \beta$-estradiol alters the activity of conventional and IFN-producing killer dendritic cells. J. Immunol. 180, 1423-1431 (2008).

40. Bengtsson, A. K., Ryan, E. J., Giordano, D. Magaletti, D. M. \& Clark, E. A. 17 $\beta$-estradiol (E2) modulates cytokine and chemokine expression in human monocyte-derived dendritic cells. Blood 104 . 1404-1410 (2004).

41. Murphy, H. S. et al. Tissue-specific effect of estradiol on endothelial cell-dependent lymphocyte recruitment. Microvasc. Res. 68, 273-285 (2004).

42. Olsen, N. J. \& Kovacs, W. J. Gonadal steroids and immunity. Endocrine Rev. 17, 369-384 (1996).

43. Rettew, J. A., Huet-Hudson, Y. M. \& Marriott, I. Testosterone reduces macrophage expression in the mouse of Toll-like receptor 4, a trigger for inflammation and innate immunity. Biol. Reprod. 78, 432-437 (2008)

44. Cutolo, M. et al. Synovial fluid estrogens in rheumatoid arthritis. Autoimmun. Rev. 3 193-198 (2004).

45. Folomeev, M. et al. Plasma sex hormones and aromatase activity in tissues of patients with systemic lupus erythematosus. Lupus 1, 191-195 (1992).

46. Sicotte, N. L. et al. Testosterone treatment in multiple sclerosis: a pilot study. Arch. Neurol. 64, 683-688 (2007).

47. Kim, K. S., Hufnagel, G., Chapman, N. M. \& Tracy, S. The group B coxsackieviruses and myocarditis. Rev. Med. Virol. 11, 355-368 (2001).
48. Mendelsohn, M. E. \& Karas, R. H. The protective effects of estrogen on the cardiovascular system N. Engl. J. Med. 340, 1801-1811 (1999).

49. Frisancho-Kiss, S. et al. Sex differences in coxsackievirus B3-induced myocarditis: IL-12Rß1 signaling and IFN- $\gamma$ increase inflammation in males independent from STAT4. Brain Res. 1126, 139-147 (2006)

50. Frisancho-Kiss, S. et al. Cross-regulation by TLR4 and $T$ cell Ig mucin-3 determines sex differences in inflammatory heart disease. J. Immunol. 178 , 6710-6714 (2007)

51. Klein, S. Host factors mediating sex differences in viral infection. Gend. Med. 2, 197-207 (2005)

52. Hannah, M. F., Bajic, V. B. \& Klein, S. L. Sex differences in the recognition of and inate antiviral responses to Seoul virus in Norway rats. Brain Behav. Immun. 22, 503-516 (2008).

53. Klein, S. L. \& Calisher, C. H. Emergence and persistence of hantaviruses. Curr. Top. Microbiol. Immunol. 315, 217-252 (2007).

54. Klingstrom, J., Lindgren, T. \& Ahlm, C. Hantavirus infection induces higher plasma levels of IL-9, FGF-2 and GM-CSF, and lower levels of IL-8 and IP-10 in females compared to males. Clin. Vaccine Immunol. 15, 885-887 (2008)

55. Barna, M., Komatsu, T., Bi, Z. \& Reiss, C. S. Sex differences in susceptibility to viral infection of the central nervous system. J. Neuroimmunol. 67 31-39 (1996).

56. Anker, $\mathrm{M}$. Addressing sex and gender in epidemicprone infectious disease [online], http://www.who.int/ csr/resources/publications/ sexandgenderinfectiousdiseases/en (2007).

57. Phuong C et al Clinical diagnosis and assessment of severity of confirmed dengue infections in Vietnamese children: is the World Health Organization classification system helpful? Am. J. Trop. Med. Hyg 70, 172-179 (2004).

58. Wagner, H. J., Hornef, M., Teichert, H. M. \& Kirchner, $\mathrm{H}$. Sex difference in the serostatus of adults to the Epstein-Barr virus. Immunobiol. 190, 424-429 (1994)

59. Villacres, M. C., Longmate, J., Auge, C. $₫$ Diamond, D. J. Predominant type 1 CMV-specific memory T-helper response in humans: evidence for gender differences in cytokine secretion. Hum. Immunol. 65, 476-485 (2004).

60. Stanberry, L. R. et al. GlaxoSmithKline herpes vaccine efficacy study group: glycoprotein-D-adjuvant vaccine to prevent genital herpes. N. Engl. J. Med. 347 1652-1661 (2002).

61. Benki, S. et al. Cyclic shedding of HIV-1 RNA in cervical secretions during the menstrual cycle. J. Infect. Dis. 189, 2192-2201 (2004).

62. MacDonald, E. M. et al. Susceptbility of human female primary genital epithelial cells to herpes simplex virus, type-2, and the effect of TLR3 ligand and sex hormones on infection. Biol. Reprod. 77 1049-1059 (2007).

63. Prins, M., Meyer, L. \& Hessol, N. A. Sex and the course of HIV infection in the pre- and highly active antiretroviral therapy eras. AIDS $19,357-370$ (2005).

64. Institute of medicine committee on understanding the biology of sex and gender differences, board on health science policy in Exploring the Biological Contributions to Human Health: Does Sex Matter? (eds Wizemann, T. M. \& Pardue, M.-L.) 13-228 (National Academies Press, Washington DC, 2001).

65. Aaby, P. Is susceptibility to severe infection in lowincome countries inherited or acquired? J. Intern. Med. 261, 112-122 (2007)

66. Palaszynski, K. M. et al. A ying-yang effect between sex chromosome complement and sex hormones on the immune response. Endocrinol. 146, 3280-3285 (2005).

67. Gandhi, M., Aweeka, F., Greenblatt, R. M. \& Blaschke, T. F. Sex differences in pharmacokinetics and pharmacodynamics. Annu. Rev. Pharmacol. Toxicol. 44, 499-523 (2004)

68 Ofotokun, I., Chuck, S. K. \& Hitti, J. E. Antiretroviral pharmacokinetic profile: a review of sex differences. Gend. Med. 4, 106-119 (2007).

69. Nicastri, E. et al. Sex issues in HIV-1-infected persons during highly active antiretroviral therapy: a systematic review. J. Antimicrob. Chemother. 60 724-732 (2007)

70. Hoffman, R. M., Umeh, O. C., Garris, C., Givens, N. \& Currier, J. S. Evaluation of sex differences of fosamprenavir (with and without ritonavir) in HIV-infected men and women. HIV Clin. Trials 8 , 371-380 (2007)

71. Collazos, J., Asensi, V. \& Carton, J. A. Sex differences in the clinical, immunological and virological parameters of HIV-infected patients treated with HAART. AIDS 21, 835-843 (2007).

\section{Acknowledgements}

The author acknowledges the Canadian government for the award of a Canada Research Chair in Women's Health \& Immunonbiology, and Gillian Einstein (Department of Psychology, University of Toronto) for critical review of the manuscript.

\section{DATABASES}

Entrez Gene: http://www.ncbi.nlm.nih.gov/entrez/query. fcgi?db=gene

CCR5 $|\underline{\text { CD16 }}| \underline{\text { ER } \alpha}|\underline{\text { ER } \beta}|$ FOXP3 $|\underline{\text { IL-6 }}|$ IRF1 $\mid$ TIM3 $\mid$ INF OMIM: http://www.ncbi.nlm.nih.gov/entrez/query. fcgi?db=OMIM

ankylosing spondylitis | Goodpasture syndrome |Graves' disease | Hashimoto's thyroiditis |multiple sclerosis $\mid$ systemic lupus erythematosus $\mid \underline{X}$-linked severe combined immunodeficiency

FURTHER INFORMATION

E. N. Fish's homepage: http://www.oci.utoronto.ca/ researchers/profile.php?lookup=1831 WHO page on sexually transmitted infections: http://www. who.int/mediacentre/factsheets/fs110/en

ALL LINKS ARE ACTIVE IN THE ONLINE PDF 